\title{
AUTOMATED CUTTING TOOLS SELECTION FOR BEARINGS COMPONENTS TURNING PROCESS
}

\author{
DAN GEORGE PRUTICA ${ }^{* 1}$, GHEORGHE BRABIE ${ }^{1}$, BOGDAN CHIRITA ${ }^{1}$ \\ I "Vasile Alecsandri" University of Bacau, Calea Marasesti 157, Bacau, 600115, Romania
}

\begin{abstract}
Due to the high potential of improving efficiency and productivity in machining operations, the automated cutting tools selection and the optimization of cutting parameters has been an area of high interest for many researchers in the frame of CAPP systems development. Through the work presented in this paper an automated cutting tools selection algorithm for roughing and finishing turning has been realized. An example for turning of the outer rings for large-size single row cylindrical roller bearings has been presented to illustrate the application of the proposed algorithm and analyze the results.
\end{abstract}

Keywords: automated cutting tools selection, turning process, computer aided process planning (CAPP), bearings manufacturing

\section{INTRODUCTION}

Process planning is a complex multi-task consisting in selecting the necessary processes, tools, optimum parameters and operation sequencing for the transformation plan of a product idea, from the design phase, into a physical product, in the manufacturing phase. The selection of cutting tools and the calculation of cutting parameters is a sub-function of the manufacturing process planning requiring extensive knowledge and considerable experience affecting directly the product cost [1-4].

Traditionally the process planning is done by experienced planners, usually being time consuming and depending on the planner experience. The use of computer technology for process planning was initiated more than fifty years ago being known as Computer Aided Process Planning (CAPP). Due to CAPP importance in manufacturing for improving efficiency and productivity in machining operations, the automated cutting tools selection and the optimization of cutting parameters has been an area of high interest for many researchers in the frame of CAPP systems development [5-13].

\section{CUTTING TOOLS SELECTION ALGORITHM}

\subsection{Product design and process planning knowledge-based system overview}

Through the work from this paper the authors are presenting the automated cutting tools selection module of a knowledge-based CAPP system for bearings components turning process. The automated cutting tools selection module is integrated with a cutting parameters optimization module presented by the authors in a separate paper.

A knowledge-based CAPP system incorporates the manufacturing knowledge data containing all the constraints and rules necessary for making optimal decisions to generate product process plans. The overview of the Product Design and Process Planning Knowledge-based system for bearings components turning process is presented in

\footnotetext{
* Corresponding author, email: prutidng@ schaeffler.com

(C) 2020 Alma Mater Publishing House
} 
Figure 1, containing a system framework, integrated sections for product design and process planning, connected data bases and connected commercial CAD/CAM system. The process planning system section includes all the required functionality for automated generation of process plans for bearings components manufacturing, structured on modules for part feature recognition, generation of manufacturing features, technology selection, machine selection, clamping methods selection, tools selections, process parameters optimization, and machining sequence generation. The manufacturing knowledge data is introduced and maintained within the system by planning specialists. The raw material data, machine data and tools data are stored in specific databases connected to the system $[12,14]$.

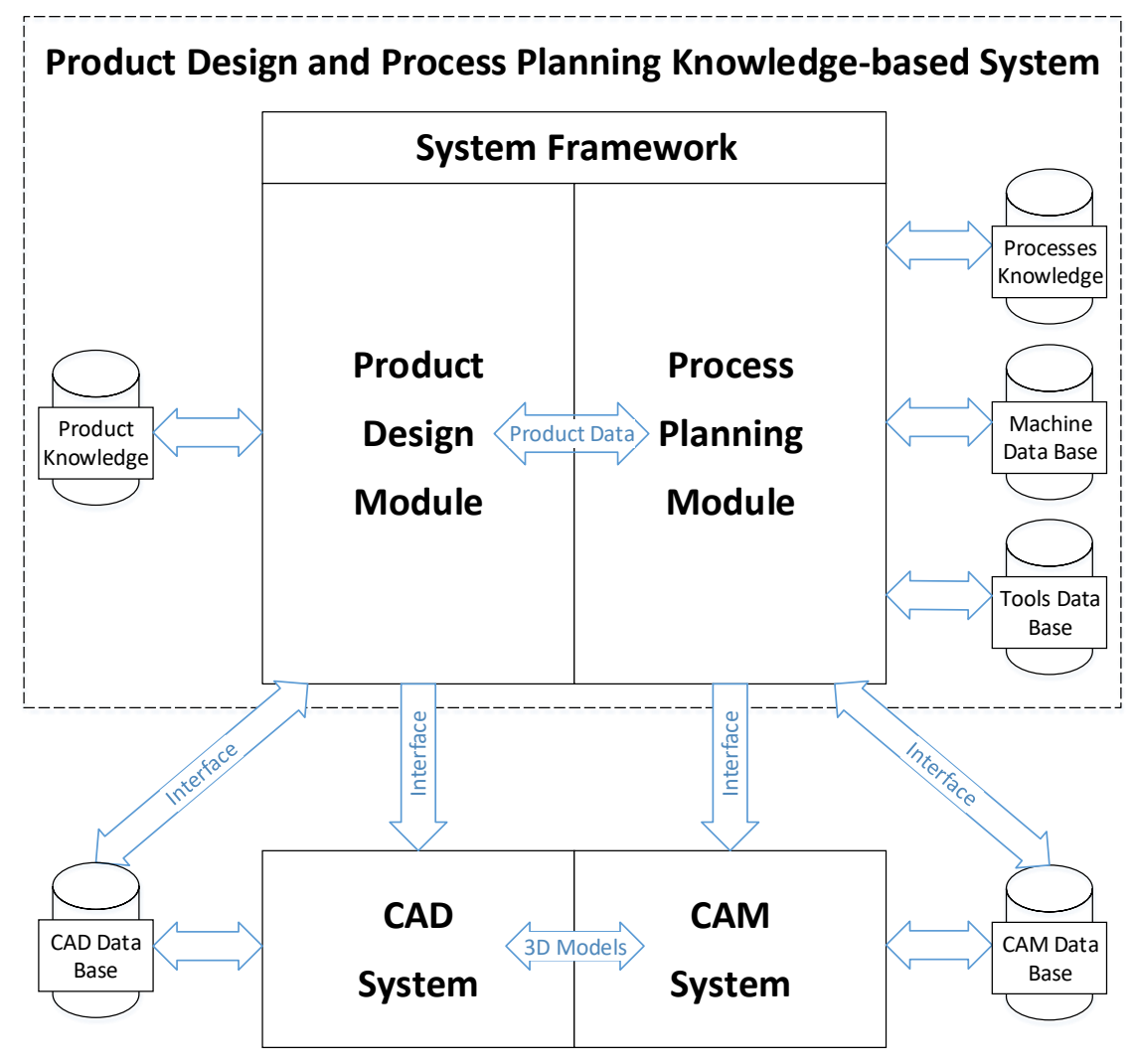

Fig. 1. Product Design and Process Planning Knowledge-based System [12].

\subsection{Input data for cutting tools selection}

The required input data for cutting tools selection are the workpiece material and geometry, part geometry and required surface finish, available machine characteristics and available tools characteristics. The data related to the workpiece and part are provided by the connected sections of the Product Design and Process Planning Knowledge-based system for bearings and the data related to machine and tools are available in the dedicated data bases connected to the system. In this paper, data from the tool manufacturer Sandvik is considered as an example.

Bearings components are in general parts composed from a combination of predefined design features selected and calculated to form the finished part, offering a high potential for applying a form of design by features approach for the feature recognition in the integration between CAD and CAPP. The section for product design of the Product Design and Process Planning Knowledge-based system has been previously developed to calculate and generate the bearings design configurations. The generated part configuration contains the design features of the bearings components which will be used as input data for the process planning section. The process planning module for part feature recognition is generating the corresponding manufacturing features, based on a set of rules for conversion, according to the specific characteristics of the design features, process technology and machines specific capabilities.

\subsection{Cutting tools selection algorithm}

For the work presented in this paper the objective is to minimize the machining time of each operation respecting the constrain for the number of tools, which should not exceed the maximum available number of tools in turret. 
Cutting tools sets are selected depending mainly on the workpiece material, raw material delivery conditions, manufacturing feature, operation type and cost criteria. The proposed algorithm in the current work has two phases presented in Figure 2, in the first phase are selected all the possible tool sets available for each manufacturing feature and in the second phase is performed the final selection of the optimal tool set for each manufacturing feature.

Phase 1

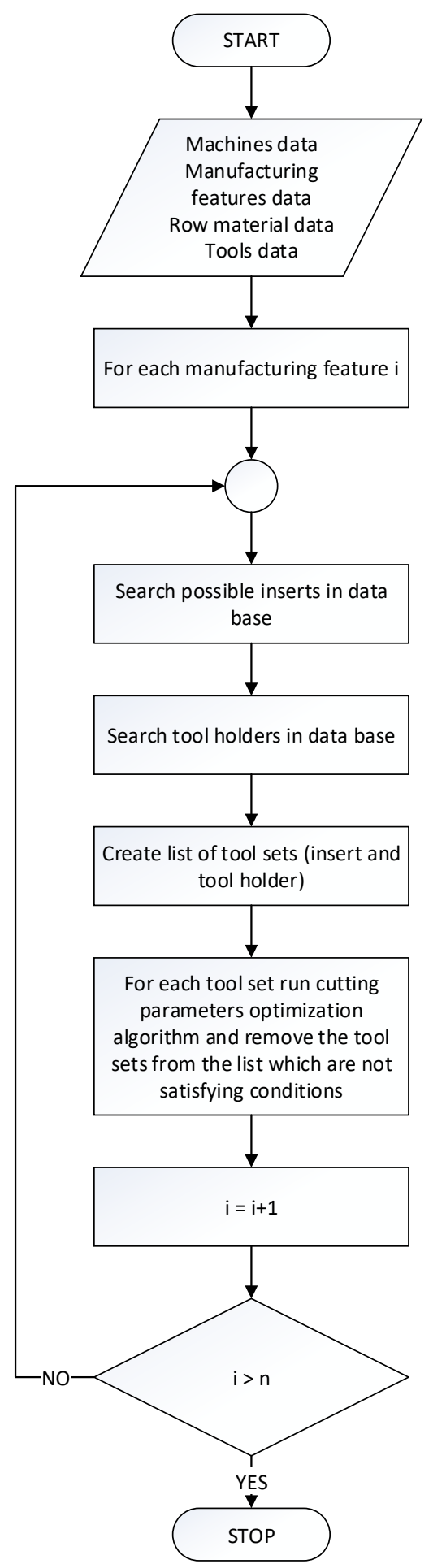

Phase 2

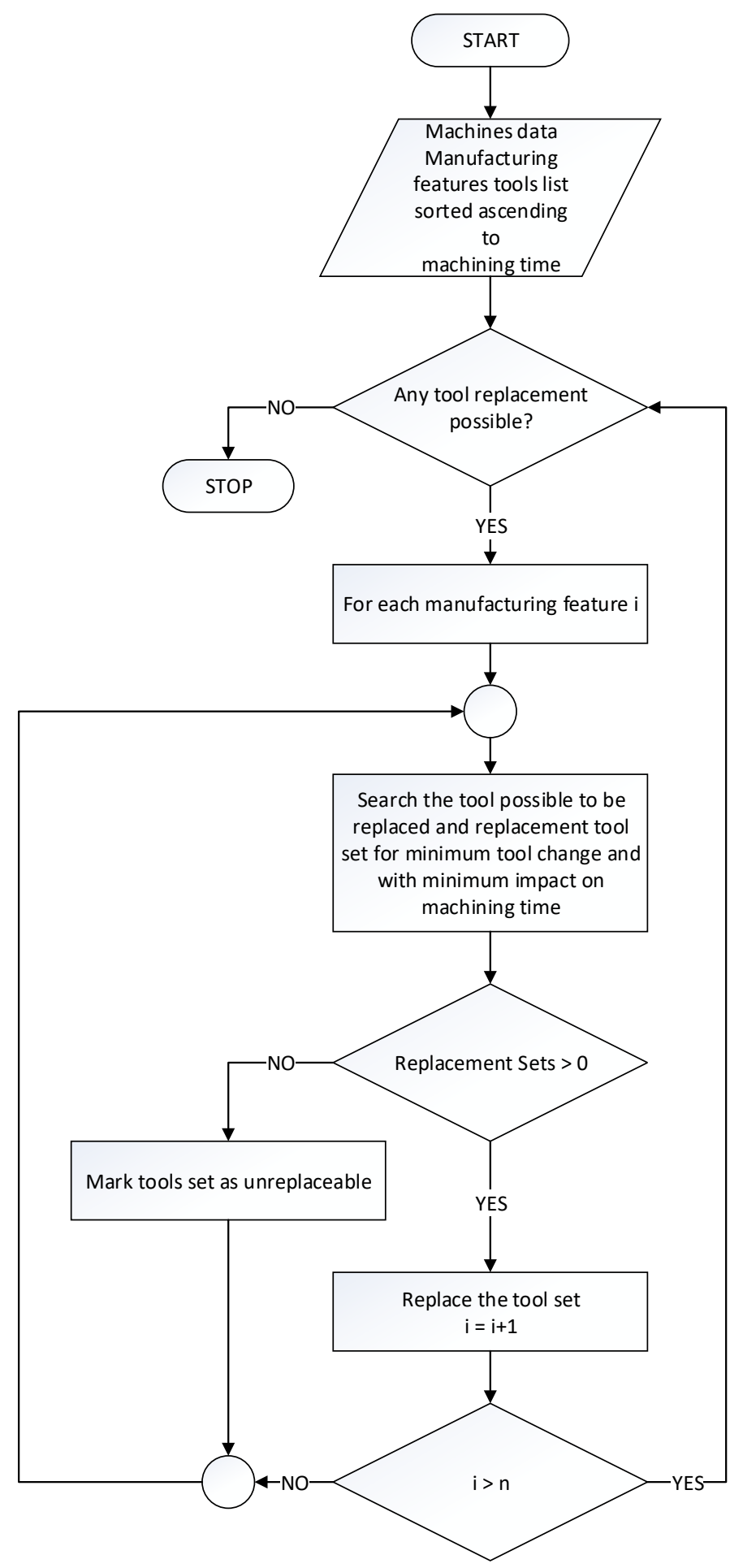

Fig. 2. First and second phase for cutting tools selection algorithm. 
The list of possible tool sets for each manufacturing feature selected in the first phase of the algorithm, is created by selecting all the tool available which are satisfying the specific conditions presented in Table 1.

Table 1. Tool sets selection criteria.

\begin{tabular}{|c|c|}
\hline Criteria category & Criteria \\
\hline \multirow{3}{*}{ Material } & Workpiece material groups: $\mathrm{P}, \mathrm{M}, \mathrm{K}, \mathrm{N}, \mathrm{S}$ and $\mathrm{H}$ \\
\hline & Chip breaking \\
\hline & Hardness \\
\hline \multirow{5}{*}{ Component } & Operation: roughing, medium or finishing \\
\hline & Type of operation: longitudinal, facing and profiling \\
\hline & Operational versatility: small, medium and high \\
\hline & Feature position: external or internal \\
\hline & Feature position relative to part section: left, right or between \\
\hline \multirow{6}{*}{ Machine } & Stability, power and torque \\
\hline & Component clamping \\
\hline & Tool position \\
\hline & Tool changing times \\
\hline & Number of tools in turret \\
\hline & Spindle speed (rpm) \\
\hline
\end{tabular}

The cutting parameters and the machining time, for each possible tool sets from the list, are calculated with the cutting parameters optimization module previous developed. When a tool set is not satisfying the conditions defined in the cutting parameters algorithm will be removed from the tool list.

In the second phase of the algorithm is performed an optimization of the tool sets selection having as objective minimum tool change with minimum impact on machining time which will lead to a minimum number of selected tool sets. This phase strongly depends on the operations sequences determined in the dedicated module of the knowledge-based CAPP system for bearings components turning process.

\section{RESULTS AND DISCUSSION}

Several tests were conducted to validate the results of the tools selection algorithm. Two representative types, NU and $\mathrm{N}$ type, of large-size cylindrical rollers bearings were selected as examples. The selected bearings outer rings have a wide range of dimensions as it can be observed from the data presented in Table 2. The selected turning machines are Hessapp VDM 1200 for bearing NU10/560-TB-M1 and Hessapp DVT 750 for bearing N334-E-TBM1. Each machine has the capability of simultaneous turning with two machine heads equipped with turrets having eight tool pockets.

Table 2. Main dimensions of the selected bearings outer rings, Source: Schaeffler.

\begin{tabular}{|l|l|l|}
\hline & \multicolumn{1}{|c|}{ NU } \\
\hline Bearing Type & \multicolumn{2}{|c|}{} \\
\hline $\begin{array}{l}\text { Bearing catalogue } \\
\text { designation }\end{array}$ & $\mathrm{NU}$ \\
\hline Outer Diameter (D) & $820 \mathrm{~mm}$ & \\
\hline Width (B) & $115 \mathrm{~mm}$ & $360 \mathrm{~mm}$ \\
\hline Raceway Diameter (E) & $754 \mathrm{~mm}$ & $72 \mathrm{~mm}$ \\
\hline Board diameter (D1) & $728.4 \mathrm{~mm}$ & $318 \mathrm{~mm}$ \\
\hline
\end{tabular}


Each bearing has a specific number of design features forming their geometry and for each design feature, in the knowledge-based CAPP system, are generated corresponding manufacturing features as it can be observed from the data presented in Figure 3.

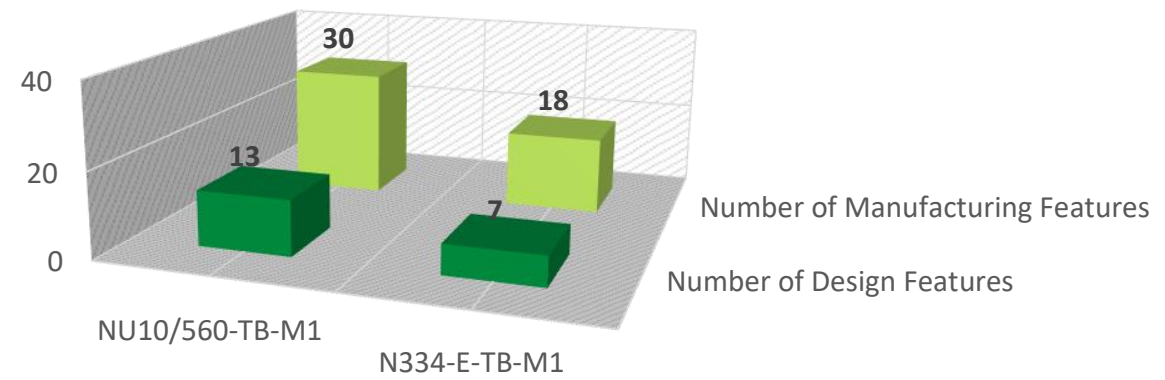

Fig. 3. Number of design features and manufacturing features for the selected bearings.

The numbers of manufacturing features allocated per each tool set for bearing NU10/560-TB-M1 are presented in Figure 4 and

As an example, in Figure 5 are illustrated the manufacturing features for roughing turning, in first and second clamping, for the outer ring of bearing NU10/560-TB-M1.

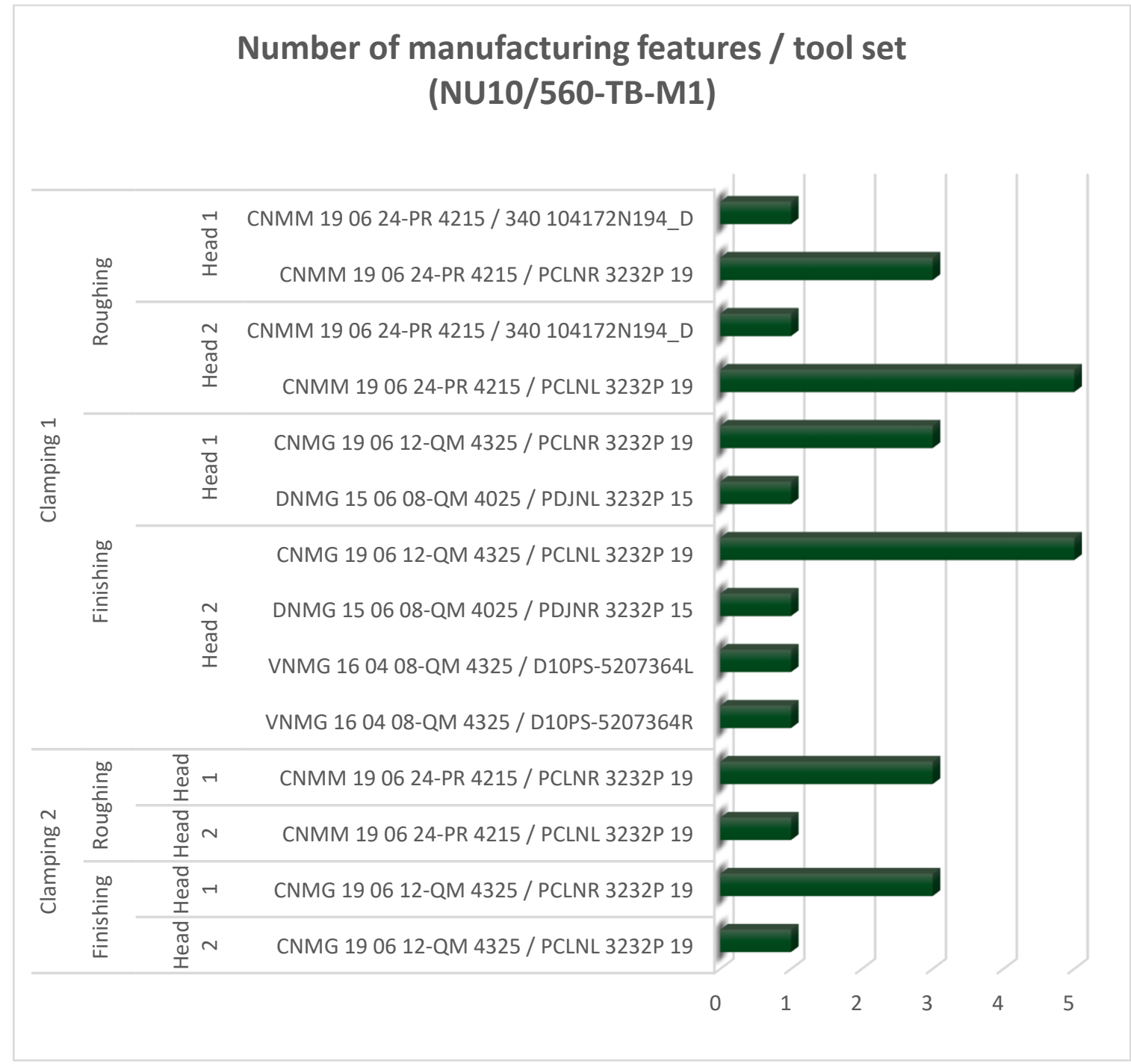

Fig. 4. Number of manufacturing features allocated per tool set for bearing NU10/560-TB-M1. 


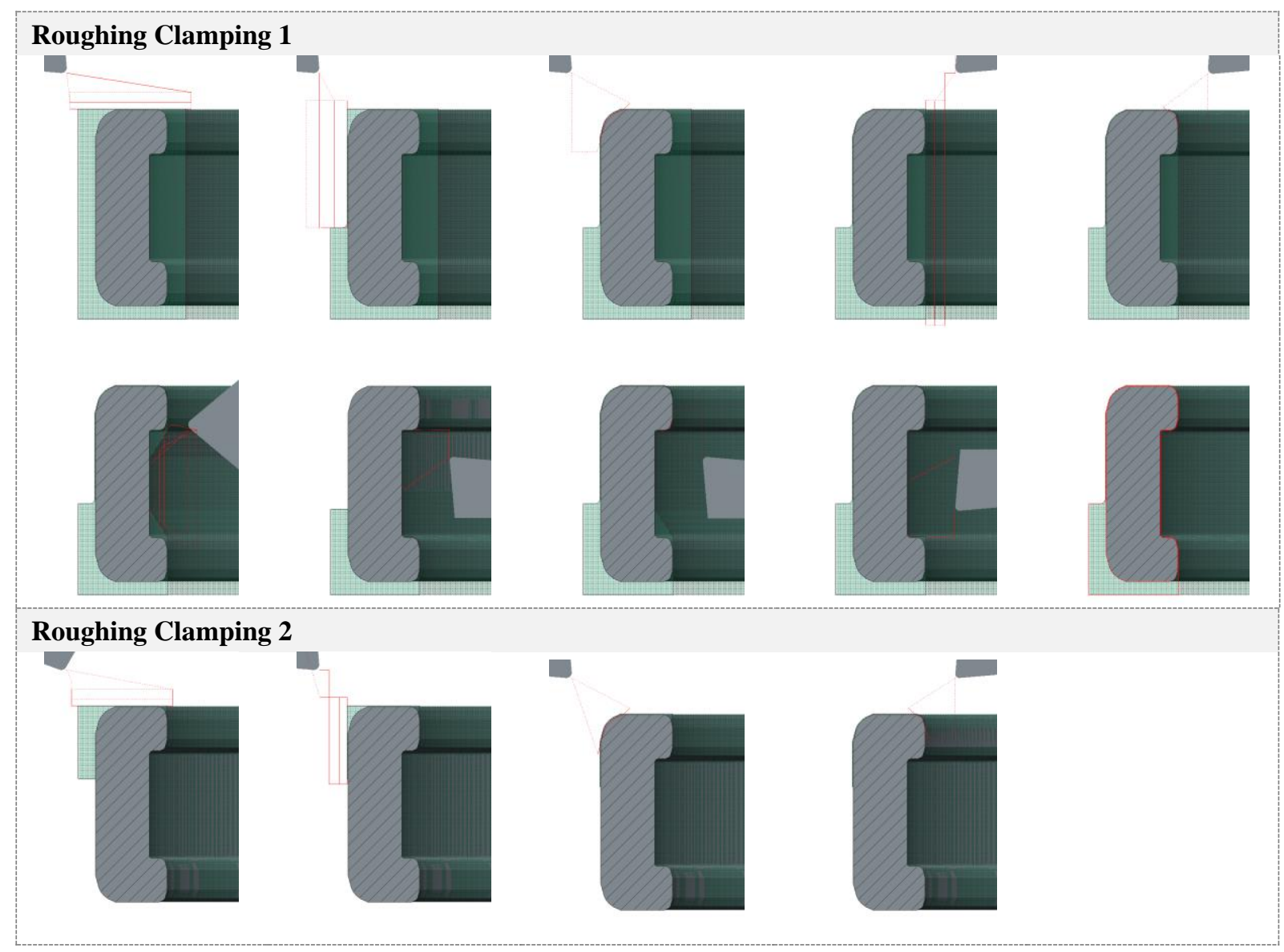

Fig. 5. Manufacturing features for roughing in first and second clamping for bearing NU10/560-TB-M1.

The numbers of manufacturing features allocated per each tool set for bearing N334-E-TB-M1 are presented in Figure 6.

\section{Number of manufacturing features / tool set (N334-E-TB-M1)}
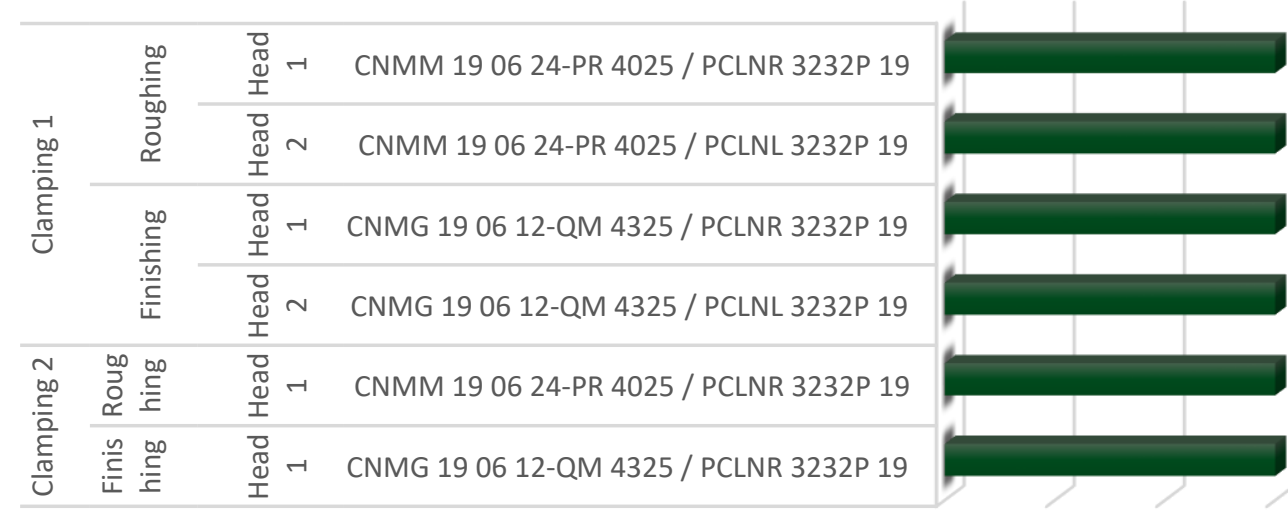

Fig. 6. Number of manufacturing features allocated per tool set for bearing N334-E-TB-M1. 
In the first example, for bearing NU10/560-TB-M1, are selected ten tool sets allocated on the two machine heads. In the first clamping all tool sets are utilized for turning of 22 manufacturing features, 10 roughing and 12 finishing and in the second clamping, six tool sets are utilized for turning of eight manufacturing features, four roughing and four finishing.

In the second example, for bearing N334-E-TB-M1, are selected four tool sets allocated on the two machine heads. In the first clamping all tool sets are utilized for turning of 12 manufacturing features and in the second clamping, two tool sets are utilized for turning of six manufacturing features.

\section{CONCLUSIONS}

The current paper presents an automated cutting tools selection algorithm for roughing and finishing turning which has been integrated as module in a knowledge-based CAPP system for bearings components turning process. The proposed algorithm has two phases, in the first phase are selected all the possible tool sets available for each manufacturing feature and in the second phase the selection of the tool sets is optimized having as objective minimum tool change with minimum impact on machining time. The automated cutting tools selection module is integrated with a cutting parameters optimization module presented by the authors in a separate paper. Examples for roughing and finishing turning have been presented to illustrate the application of the proposed algorithm and to validate the results. This method can be applied for all tools from different manufacturers, in this paper, data from the tool manufacturer Sandvik is considered as an example.

\section{REFERENCES}

[1] Jain, N.K., Jain, J.V., Process selection methodology for advanced machining processes, Journal of Advanced Manufacturing Systems, vol. 02, no. 01, 2003, p. 5-45.

[2] Cheng, K., Machining dynamics: Fundamentals, Applications and Practices, Springer London, 2009.

[3] Ridgway, K., Selection of cutting tools and conditions of machining operations using an expert system, Computers in Industry, vol. 42, no. 1, 2000, p. 43-58.

[4] Fernandes, K, Raja, R.V., Incorporated tool selection system using object technology, International Journal of Machine Tools and Manufacture, vol. 40, no. 11, 2000, p. 1547-1555.

[5] Al-wswasi, M., Ivanov, A., Makatsoris, H., A survey on smart automated computer-aided process planning (ACAPP) techniques, The International Journal of Advanced Manufacturing Technology, vol. 97, no. 1-4, 2018, p. 809-832.

[6] Wierda, L., Linking design, process planning and cost information by feature-based modelling, Journal of Engineering Design, vol. 2, no. 1, 2007, p. 3-19.

[7] Oral, A., Cakir, M.C., Automated cutting tool selection and cutting tool sequence optimisation for rotational parts, Robotics and Computer Integrated Manufacturing, vol. 20, no. 2, 2004, p. 127-141.

[8] Jaider, O., El Mesbahi, A., Rechia, A., Zarkti, H., An automatic Feature-based tool selection approach for turning process based on data from Sandvik Coromant, Xème Conférence Internationale: Conception et Production Intégrées, Tanger, Morocco, 2015

[9] Bala, M, Chang, C.T., Automatic cutter selection and optimal cutter path generation for prismatic parts, C. T., International Journal of Production Research, vol. 29, no. 11, 2007, p. 2163-2176.

[10] Raja, S., Baskar, N., Particle swarm optimization technique for determining optimal machining parameters of different work piece materials in turning operation, The International Journal of Advanced Manufacturing Technology, vol. 54, 2010, p. 445-463.

[11] Lau, H.C.W., Lee, C.K.M., Jiang, B., Hui, I.K., Pun, K.F., Development of a computer-integrated system to support CAD to CAPP, The International Journal of Advanced Manufacturing Technology, vol. 26, 2005, p. 10321042.

[12] Prutica, D.G., Brabie, G., New Approach for the Integration of CAD/CAPP/CAM Systems for Bearings Manufacturing, The 30th SIAR International Congress of Automotive and Transport Engineering. SMAT 2019. Springer, Cham, 2019.

[13] Yusof, Y., Latif, K., Survey on computer-aided process planning, The International Journal of Advanced Manufacturing Technology, vol. 75, no. 1-4, 2014, p. 77-89.

[14] https://www.sandvik.coromant.com/en-us/downloads (25.09.2019). 